\title{
How does one extend the Chart of Nuclides?
}

\author{
Gurgen Adamian, Joint Institute for Nuclear Research, Dubna, Russia \\ Nikolai Antonenko, Joint Institute for Nuclear Research, Dubna, and Tomsk Polytechnic University, Russia \\ Alexis Diaz-Torres, University of Surrey, UK \\ Sophia Heinz, GSI Helmholtzzentrum Darmstadt and Justus-Liebig-Universität Giessen, Germany
}

\begin{abstract}
Nuclear physicists all over the world are searching for new exotic nuclei. But their ambitions are being hindered by the lack of effective state-of-the-art methods for laboratory nucleosynthesis. Activities are ongoing in many places to find new pathways for production and detection of exotic nuclei. But how promising are these efforts? Here we give an overview of the ongoing worldwide activities.
\end{abstract}

\section{Objects of desire}

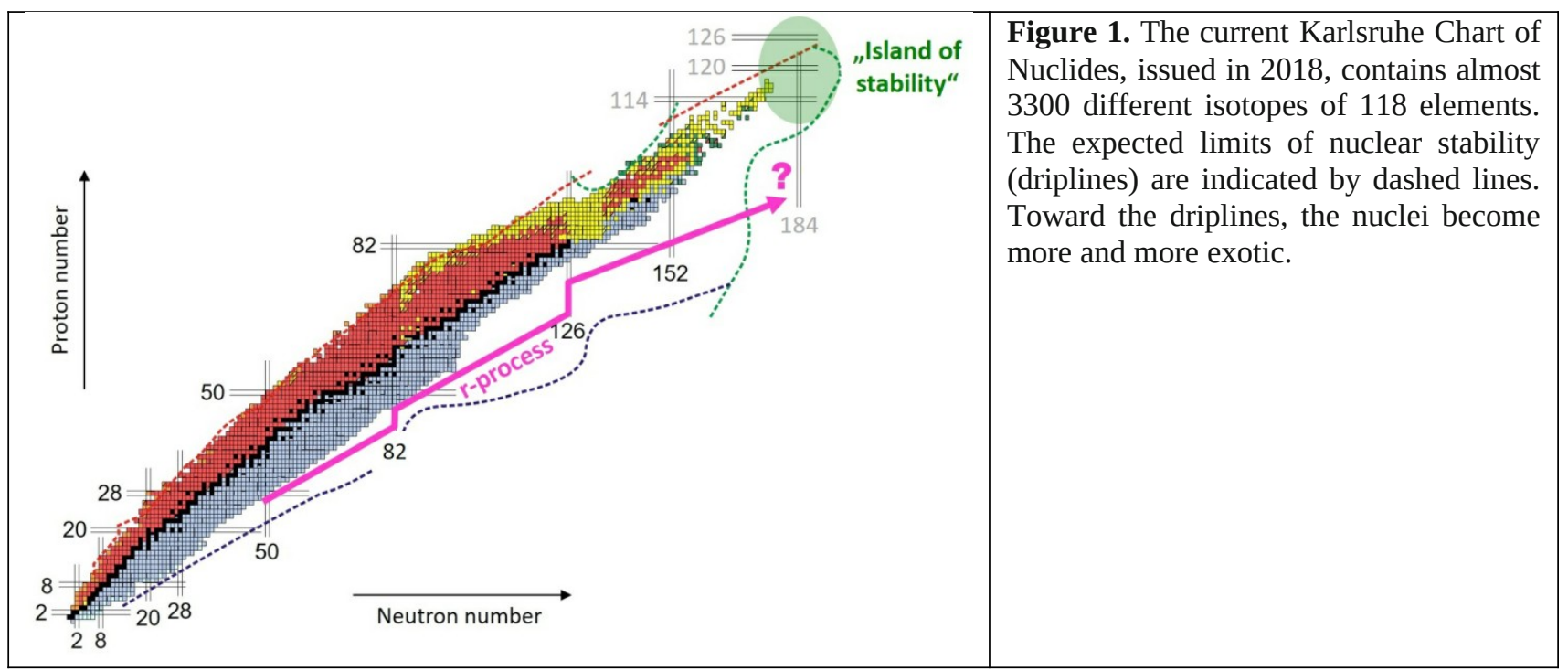

How and where are the chemical elements created in the universe? Which nuclear reactions determine the evolution and destiny of stars? And what is the nature of the still obscure nuclear force? Such fundamental questions occupy nuclear physicists. The answers are mostly hidden in the properties of exotic nuclei, like their binding energy, half-life or shape. Exotic nuclei are unstable and do not occur in our natural environment on Earth, therefore we have to produce them artificially in the lab. This is what nuclear physicists have been doing for many decades. Meanwhile, we know of the existence of more than 3000 different isotopes of 118 elements (Fig.1), with about 90 percent of them being man-made [1]. Each nuclide has its own individual combination of protons and neutrons and is governed by the sensitive interplay between the attractive nuclear force and the repulsive Coulomb force which determines its properties. Model predictions indicate that another 4000 isotopes are still awaiting their discovery, with the vastest unexplored territory located on the neutron-rich side in the upper half of the nuclide chart. Most of the astrophysical rapid neutron capture process (r-process), which is assumed to be responsible for the production of the heavy elements in stellar explosions, proceeds through this unknown territory. By studying the properties of nuclei along the r-process path we can understand the astrophysical synthesis of the heavy elements and their abundances in nature. It is still obscure where the r-process ends in the upper part of the nuclide chart. Presumably it penetrates deep into the territory of neutron-rich superheavy nuclei. New magic neutron and proton numbers are predicted in this region at $\mathrm{N}=184, \mathrm{Z}=114$ or $120-126$ creating an "island of stability". Nuclei on this "island" are expected to have higher fission barriers, resulting in an enhanced stability against fission. Is the "island of stability" the endpoint of the r-process path? 


\section{Nucleosynthesis in the Lab}

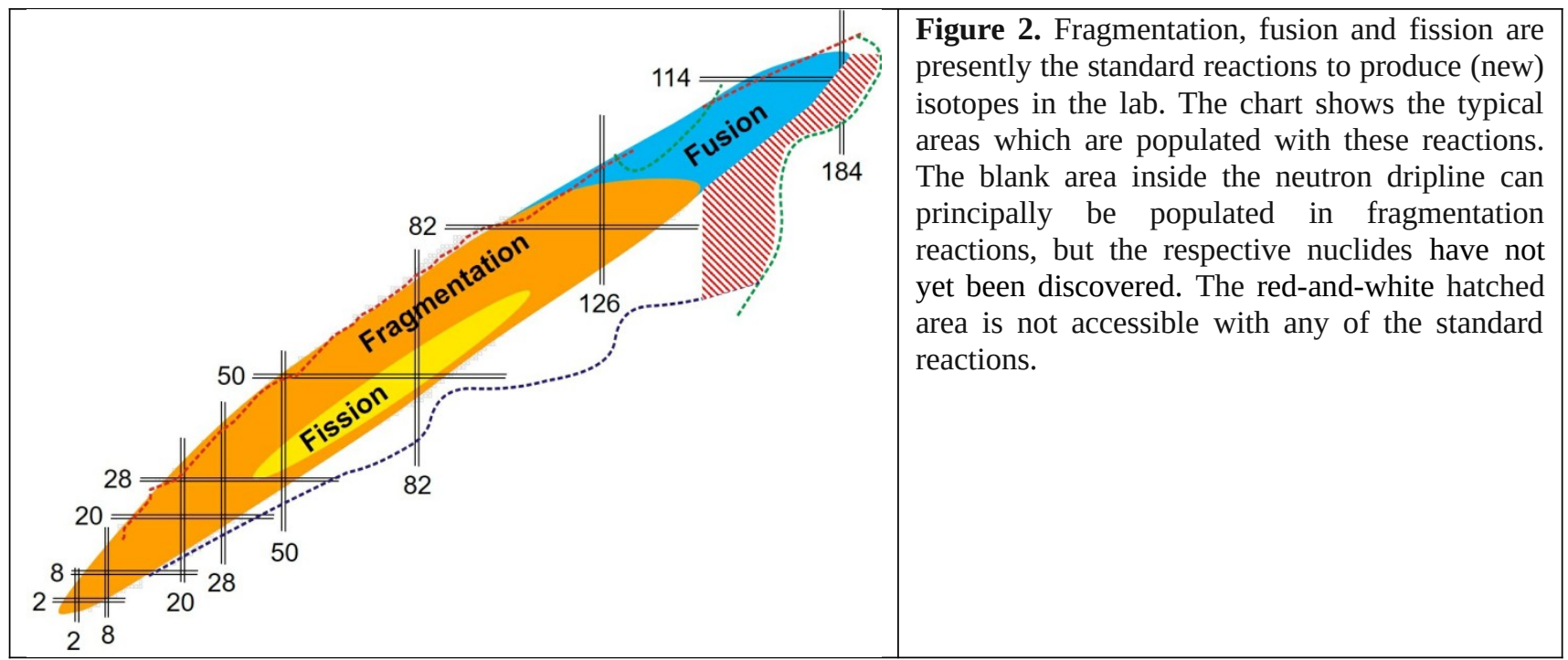

Currently, nuclear physicists are using three "standard" reactions to produce exotic nuclei in the lab: fusion, fragmentation, and fission (Fig.2). Fragmentation of heavy nuclei at relativistic energies is very efficient to produce isotopes all-over the nuclide chart, from neutron-deficient to neutron-rich. However, it is limited to elements no heavier than uranium ( $\mathrm{Z}=92)$, because uranium is the heaviest applicable beam or target material. Fission reactions lead typically to neutron-rich intermediate heavy nuclides. Fusion so far offers the only possible way to synthesize transuranium and superheavy nuclei [2, 3], but it results in rather neutron-deficient isotopes on the left side of the stability line. The capabilities of fragmentation, fusion and fission reactions determine the present limits of the nuclide chart. The smallest cross-sections which are currently accessible in these reactions are on the level of picobarns $\left(10^{-12}\right.$ barns), which results in average yields of one nucleus per day in fragmentation reactions and one nucleus per 10 days in fusion reactions. These limits originate from available beam intensities coupled with applicable target thicknesses for the respective reactions.

One approach to extend the nuclide chart is to increase beam intensities. New powerful facilities are arising in many places which will provide up to 100 times higher beam currents and enable access to new isotopes with sub-picobarn cross-sections. Most of them will use fragmentation and fission reactions. Examples of such facilities are CERN-ISOLDE, FAIR/GSI (Germany), GANIL (France), HIARF (China), SPES (Italy), RIKEN (Japan), RISP (Korea), and FRIB (USA). Also, a new superheavy element "factory" which will use fusion reactions with intense primary beams is just commencing operations at the Joint Institute for Nuclear Research (JINR) in Dubna, Russia. However, in the very neutron-rich upper corner of the nuclide chart the standard reactions fail (Fig.2). In fragmentation reactions no nuclides with more than 146 neutrons can arise, given by the neutron number of uranium, the heaviest applicable nucleus for fragmentation. Natural restrictions occur also in the transuranium and superheavy element region because fusion reactions cannot populate the neutron-rich side of the stability line.

\section{Is multi-nucleon transfer an effective pathway to new nuclides?}

Searching for solutions to advance into neutron-rich territory of heavy nuclei keeps nuclear physicists busy in labs worldwide. Their biggest hope is a reaction type called multi-nucleon transfer (MNT). It occurs in deep inelastic collisions and can be regarded as an incomplete fusion process (Fig.3a). The current model concept assumes that in collisions at Coulomb barrier energies, the projectile and target nucleus can form a moleculelike system when they come in nuclear contact. Such nuclear molecules are regarded as the first step of fusion as well as MNT reactions. Once the molecule is formed, a strong exchange of nucleons and energy starts between the reaction partners. The evolution of the molecule can either result in complete fusion, or it can break up in two fragments before the compound nucleus is formed. The emitted projectile-like and target-like fragments can be quite different from the original nuclei, and also new exotic isotopes can be among them. A 
large transfer of nucleons is still observed in collisions of two very heavy nuclei like $\mathrm{U}+\mathrm{Cm}$ which comprise nearly 200 protons and 300 neutrons. Therefore, with transfer reactions one can principally enter neutron-rich territory even in the uppermost corner of the nuclide chart.

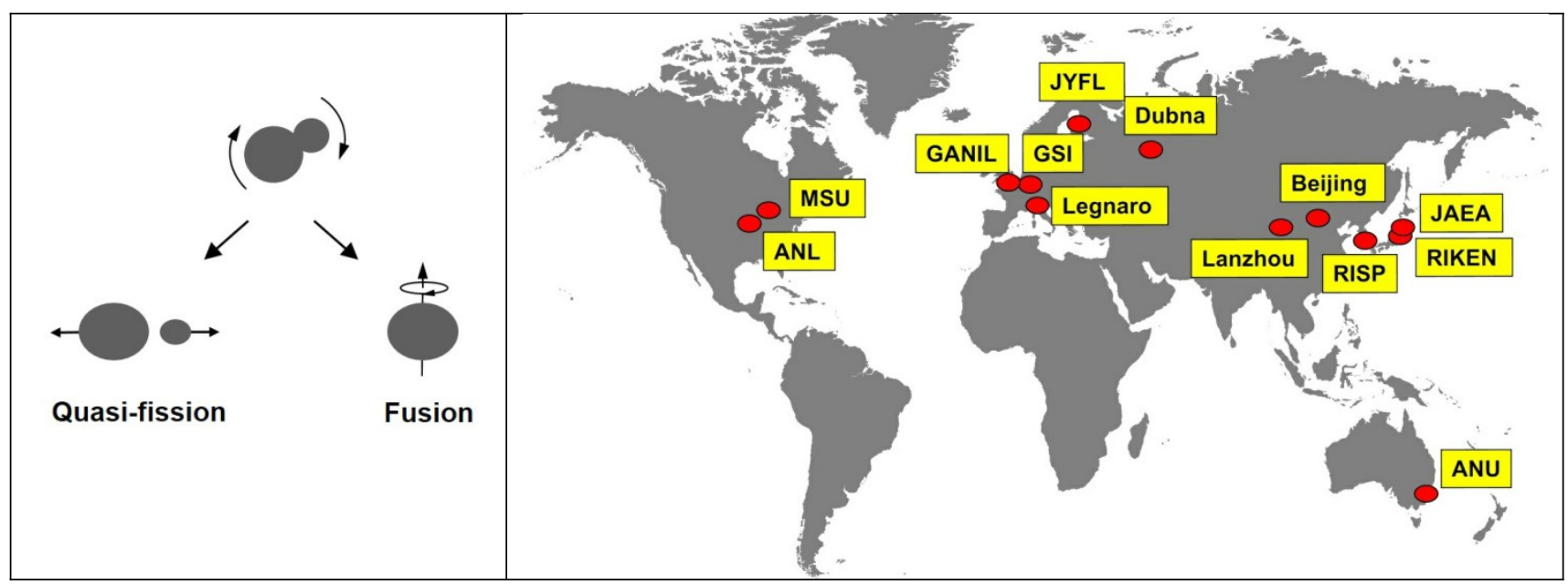

Figure 3. (a) Model concept of multinucleon transfer reactions. (b) Laboratories where MNT reactions are being investigated

MNT reactions were first observed in the late 1960s at JINR Dubna and immediately revealed their potential for nucleosynthesis [4]. A total of 75 new isotopes of elements from carbon to thorium were discovered in the years from 1970 to 1995 in Dubna, Orsay, Berkeley and at GSI, all of which are located on the neutron-rich side of the nuclide chart. Also studies of MNT reactions with the goal of synthesizing new transuranium and superheavy nuclei were performed, mainly in collisions of $\mathrm{Ca}+\mathrm{Cm}$ and $\mathrm{U}+\mathrm{Cm}$ and using radiochemical separation and detection techniques [5]. With all collision systems a vast region of heavy target-like MNT products up to mendelevium ( $\mathrm{Z}=101)$ was observed but no new transuranium or superheavy nuclei were among them. Finally, fusion reactions won the race and the application of MNT reactions for nucleosynthesis abated for the moment. Nowadays they are experiencing a revival, triggered by the necessity to uncover new territory on the nuclide chart and encouraged by promising new model calculations of MNT cross-sections [6].

There is a variety of theoretical approaches to deep inelastic collisions which can roughly be divided into macroscopic, macroscopic-microscopic, and purely microscopic models. The most wide-spread are currently the macroscopic-microscopic models which can again be divided in two main branches. One is the class of socalled dinuclear system models [7], which use diabatic internuclear potentials, while models of the other class use almost adiabatic internuclear potentials [8]. Both models involve the formation of a molecule-like nuclear system as first step of the reaction, which allows us to treat MNT and fusion reactions with a unique approach. Several degrees of freedom are used to describe the dynamics and evolution of the nuclear system. These are mainly the relative motion of the two nuclei and the mass and charge transfer between them. Furthermore, there are the deformations and orientations of the nuclei, internal excitations, among others. Different theoretical approaches indicate some common trends of MNT reactions and the magnitude of cross-sections. In general, the evolution of the nuclear molecule tends towards symmetry, meaning that nucleons move preferably from the heavy partner to the lighter one. However, to synthesize nuclei in the superheavy element region, the system must drive toward asymmetry which results in continuously decreasing cross-sections with increasing proton number of the MNT products. According to model predictions, the cross-sections of new MNT products with $Z \approx 100$ are on the order of $1 \mathrm{nb}$ and reach the $1 \mathrm{pb}$ level at $\mathrm{Z} \approx 108$. Picobarns denote the present experimental limits, meaning that MNT reactions will presumably not allow to observe new neutronrich nuclei above $\mathrm{Z} \approx 108$.

In recent years we started a new series of experiments to investigate MNT reactions in the transuranium region, mainly in collisions of ${ }^{48} \mathrm{Ca}+{ }^{248} \mathrm{Cm}$. It was performed at the velocity filter SHIP of GSI Helmholtzzentrum and enabled dropping the cross-section limit by a factor of 50 with respect to earlier 
studies. In these experiments we observed no new superheavy isotopes. The heaviest detected MNT product was the relatively neutron-rich (but already known) ${ }^{260} \mathrm{No}(\mathrm{Z}=102$ ) with a cross-section of $0.5 \mathrm{nb}$. The experiments at SHIP provided also a surprising discovery. For the first time, the neutron-deficient region around and above uranium was entered with MNT reactions, including five new uranium and transuranium isotopes around the $\mathrm{N}=126$ closed neutron shell [9]. The new nuclided reside in the only still blank area on the neutron-deficient side of the nuclide chart. So far, nuclei in this region are synthesized in fusion reactions, but the MNT data revealed that transfer reactions appear to be more efficient in synthesizing them because of the broad excitation functions of MNT products which allow to populate a wide region of nuclei in the same experiment. Fusion reactions, however, result in few selected isotopes and several experiments with different projectile-target combinations and beam energies are needed to reach the same variety of isotopes.

Model calculations (e.g. $[10,11])$ suggest to account MNT reactions also for the neutron-rich r-process region below lead. They find that MNT cross-sections overtake fragmentation cross-sections toward the neutron-rich side and seem to exceed them by many orders of magnitude when the MNT products approach the N=126 shell. Even microbarns are expected for some new isotopes. These relatively large MNT cross-sections result from the nuclear system driving toward symmetry while forming the nuclei. Most of the new-generation MNT experiments focus on this region. Studies have already been performed in several labs using beams of ${ }^{48} \mathrm{Ca}$, ${ }^{64} \mathrm{Ni}$, and ${ }^{136} \mathrm{Xe}$ on ${ }^{208} \mathrm{~Pb}$ and ${ }^{198} \mathrm{Pt}$ targets (see overview in [6]). So far, no new isotopes have been identified in this area, but first results indeed seem to confirm the model predictions.

\section{How does one identify neutron-rich heavy nuclei?}

This question seriously occupies nuclear physicists, because the lack of sensitive separation and detection techniques for heavy MNT products is an important bottleneck which hinders the entering of neutron-rich territory. The desired MNT products are heavy and slow, they have wide angular and energy distributions, they are beta-emitters, and their expected cross-sections are on the sub-microbarn scale. These properties make their separation from background events and isotope identification a "challenge". There are different approaches to separate MNT products. With radiochemical techniques nuclei from a wide angular range are stopped in a catcher foil or gas catcher, followed by separation of different chemical elements with chemical techniques. After separation, samples are prepared for isotope identification by decay tagging. Cross-sections down to $20 \mathrm{nb}$ were reached. The minimum time for one measurement cycle is about one minute. Therefore the method is not applicable for nuclei with shorter half-lives like it is the case for neutron-rich isotopes below lead. The other approach is in-flight separation and successive isotope identification by decay tagging in the focal plane of the separator. In-flight separation is fast and allows detection of nuclei down to microsecond half-lives, limited by the typical flight times of the reaction products through the separator. In-flight separators accept only reaction products emitted to forward angles which leads to large losses of MNT products. In our experiments we use the velocity filter SHIP of GSI as in-flight separator which lead to cross-section limits of $0.5 \mathrm{nb}$.

The quite high sensitivity of both separation techniques relies on isotope identification by alpha decay tagging. The signature of an alpha-decay chain is mostly so clear that a single chain is sufficient to pin down the isotope. This method works excellently in the neutron-deficient transuranium and superheavy element region but it fails for neutron-rich MNT products which are $\beta$-emitters. Principally, they can be identified by gamma rays which follow the $\beta$-decay but the method does not allow for detection of MNT products with cross-sections below microbarns.

Labs worldwide are currently investigating detection techniques which do not depend on the radioactive decays of the MNT products. The universal standard technique to determine A and Z by measuring time-offlight, kinetic energy and energy loss of the nuclei works only for MNT products with masses up to A 150. But for very heavy and slow nuclei like MNT and fusion products, the pulse height deficit deteriorates energy resolution, and with this A and Z resolution. Another approach is followed by RIKEN (Japan), JINR Dubna and Argonne National Lab who stop the MNT products in a gas catcher and apply laser resonance ionization inside the catcher to determine the atomic number $\mathrm{Z}$ [12]. After extraction from the catcher, the ions are passing a magnetic dipole field for A/q separation which reveals the mass number A of the singly charged ions. The RIKEN setup with the name KISS (KEK Isotope Separation System) is already in operation and 
first studies have been performed. The overall efficiency of the setup is on the level of $0.1 \%$. The method is suitable for ions with lifetimes of several $100 \mathrm{~ms}$ or longer, given by the extraction time from the gas catcher.

At the Fragment-Separator Facility of GSI a method is developed to identify heavy MNT products by highprecision mass measurements with a multiple reflection time-of-flight mass spectrometer (MR-TOF-MS) [13]. The production target is installed inside an ion catcher, where the MNT products are stopped after creation. Then, they are extracted from the ion catcher and injected to the MR-TOF-MS to measure their mass. The method allows for the detection of nuclei with half-lives longer than $10 \mathrm{~ms}$ and cross-sections of $100 \mu \mathrm{b}$ or more.

\section{What can we expect?}

With these efforts, how many new transuranium and superheavy nuclei can we expect in the near and midterm future? We estimate that MNT reactions can provide roughly 150 new transuranium isotopes with proton numbers up to $Z \approx 108$, the majority of them on the neutron-deficient side. Toward the neutron-rich side, one can realistically expect new isotopes of elements with proton numbers $Z \leq 108$ and neutron numbers next to the already known ones. Not to forget, in the superheavy element region at $104 \leq \mathrm{Z} \leq 108$ we expect still about 50 new isotopes from "conventional” fusion reactions with stable beams and actinide targets [6]. They will fill the present gap between the regions of cold and hot fusion residues. Altogether it results in a total of about 200 new transuranium and superheavy nuclides which is a good number compared to the 348 presently known isotopes in this area. The still empty region which is not accessible over the long term is the predicted "island of stability”. MNT reactions of two neutron-rich nuclei, or fusion reactions with neutron-rich radioactive ion beams would be the only approaches to this territory, but the intensities of radioactive beams needed are far too small for experimental realization.

For new neutron-rich nuclei below ${ }^{208} \mathrm{~Pb}$, fragmentation and possibly MNT reactions will remain powerful sources for new isotopes, particularly at the new facilities for exotic ions which are presently emerging in many places. Projectile fragmentation is still unrivalled in terms of the effectiveness of isotope separation and identification. On the other hand, there are strong indications that MNT cross-sections exceed fragmentation cross-sections by many orders of magnitude toward smaller $\mathrm{Z}$ and larger $\mathrm{N}$ of the reaction products. Future experiments will shed more light on the situation and one can be curious as to whether fragmentation or MNT will finally dominate in this region.

The authors are grateful to the PhD students, Rafael Van den Bossche and Terence Vockerodt, for a careful reading of the paper and English language corrections.

[1] M. Thoennessen, The Discovery of Isotopes, A Complete Compilation (Springer International Publishing, Cham, 2016)

[2] S. Hofmann and G. Münzenberg, Rev. Mod. Phys. 72, 733 (2000)

[3] Yu.Ts. Oganessian and V.K. Utyonkov, Rep. Prog. Phys. 78, 036301 (2015)

[4] V.V. Volkov, Phys. Rep. 44, 93 (1978)

[5] M.M. Fowler et al., Radiochim. Acta 40, 75 (1986)

[6] G.G. Adamian, N.V. Antonenko, A. Diaz-Torres, and S. Heinz, Eur. Phys. J. A 56, 47 (2020)

[7] G.G. Adamian, N.V. Antonenko, A.S. Zubov, Phys. Rev. C 71, 034603 (2005)

[8] V.V. Saiko, A.V. Karpov, Phys. Rev. C 99, 014613 (2019)

[9] H.M. Devaraja, S. Heinz, et al., Phys. Lett. B 748, 199 (2015)

[10] M.-H. Mun, G.G. Adamian, N.V. Antonenko, Y. Oh, and Y. Kim, Phys. Rev. C 89, 034622 (2014)

[11] A.V. Karpov and V.V. Saiko, Phys. Rev. C 96, 024618 (2017)

[12] S. Kimura, H. Ishiyama, et al., Nucl. Instrum. Methods B 376, 338 (2015)

[13] T. Dickel et al., Nucl. Instrum. Methods A 777, 172 (2015) 